\title{
Early laboratory values after liver transplantation are associated with anastomotic biliary strictures
}

\author{
Matthew Fasullo', Priyanush Kandakatla ${ }^{3}$, Reza Amerinasab ${ }^{3}$, Divyanshoo Rai Kohli ${ }^{4}$, Tilak Shah², \\ Samarth Patel ${ }^{2}$, Chandra Bhati ${ }^{5}$, Doumit Bouhaidar ${ }^{1}$, Mohammad S. Siddiqui ${ }^{1, *}$, Ravi Vachhani ${ }^{1, *}$ \\ 'Division of Gastroenterology, Hepatology, and Nutrition, Virginia Commonwealth University Medical Center, Richmond, VA, United States, \\ ${ }^{2}$ Division of Gastroenterology and Hepatology, Hunter Holmes McGuire VA Medical Center, Richmond, VA, United States, \\ ${ }^{3}$ Department of Radiology, Virginia Commonwealth University Medical Center, Richmond, VA, United States, \\ ${ }^{4}$ Division of Gastroenterology and Hepatology, Kansas City VA Medical Center, Kansas City, MO, United States, \\ ${ }^{5}$ Department of Transplant Surgery, Virginia Commonwealth University Medical Center, Richmond, VA, United States
}

\begin{abstract}
Backgrounds/Aims: The aim of this study was to evaluate longitudinal changes of post-liver transplantation (LT) biliary anatomy and to assess the association of increased laboratory values after LT with the development of post-LT anastomotic biliary stricture (ABS). Methods: Adult deceased donor LT recipients from 2008 and 2019 were evaluated. ABS was defined after blinded review of endoscopic cholangiograms. Controls were patients who underwent LT for hepatocellular carcinoma who did not have any clinical or biochemical concerns for ABS.

Results: Of 534 patients who underwent LT, 57 patients had ABS and 57 patients served as controls. On MRI, ABS patients had a narrower anastomosis $(2.47 \pm 1.32 \mathrm{~mm}$ vs. $3.38 \pm 1.05 \mathrm{~mm} ; p<0.01)$ and wider bile duct at 1 -cm proximal to the anastomosis $(6.73 \pm 2.45$ $\mathrm{mm}$ vs. $5.66 \pm 1.95 \mathrm{~mm} ; p=0.01)$ than controls. Association between labs at day 7 and ABS formation was as follows: aspartate aminotransferase hazard ratio (HR): 1.014 ; $95 \%$ confidence interval (CI): 1.008-1.020, $p=0.001$; total bilirubin HR: 1.292, 95\% CI: $1.100-1.517$, $p=0.002$; and conjugated bilirubin HR: $1.467,95 \%$ CI: $1.216-1.768, p=0.001$. Corresponding analysis results for day 28 were alanine aminotransferase HR: 1.004, 95\% CI: 1.002-1.006, $p=0.001$; alkaline phosphatase HR: 1.005, 95\% CI: 1.003-1.007, $p=0.001$; total bilirubin HR: 1.233, 95\% CI: 1.110-1.369, $p=0.001$; and conjugated bilirubin HR: 1.272, 95\% CI: 1.126-1.437, $p=0.001$.
\end{abstract}

Conclusions: Elevation of laboratory values early after LT is associated with ABS formation.

Key Words: Liver transplantation; Common bile duct; Cholangiopancreatography, endoscopic retrograde; Bile ducts; Magnetic resonance imaging

\section{INTRODUCTION}

Received: July 6, 2021, Revised: September 21, 2021,

Accepted: September 29, 2021

Corresponding author: Matthew Fasullo

Division of Gastroenterology, Hepatology, and Nutrition, Virginia

Commonwealth University Medical Center, 1200 E. Broad St., Richmond,

VA 23298, United States

Tel: +1-804-828-4060, Fax: +1-804-828-5304,

E-mail: matthewj.fasullo@gmail.com

ORCID: https://orcid.org/0000-0003-3787-7626

*These authors contributed equally to this study.
Biliary strictures are the most common adverse event associated with liver transplantation (LT) [1]. Post-LT strictures have an overall incidence of $13 \%$. They are associated with increased rates of allograft rejection, allograft failure, infections, need for recurrent endoscopic therapy, and readmissions [2,3]. Anastomotic biliary stricture (ABS) is the most common type of stricture. It is presumed to result from fibrosis at the site of anastomosis $[4,5]$.

Despite the deleterious impact of ABS on outcomes, it is challenging to detect ABS after transplantation. Elevation in liver associated enzymes immediately after LT is often non-specific. Cross-sectional imaging with magnetic resonance imaging (MRI) or magnetic resonance cholangio-pancreatography (MRCP) has sub-optimal diagnostic accuracy [6] largely due to a limited 
understanding of what would be considered a normal biliary anatomy following LT. These limitations in our understanding of post-LT strictures have hampered the ability of current radiographic and laboratory values to readily detect and differentiate biliary strictures from other post-LT complications [7].

The objective of the present study was to determine if changes in laboratory values in the early post-transplant timeframe could predict the later development of ABS. We also sought to define longitudinal changes of post-LT biliary anatomy. We hypothesized that the development of ABS could be detected in the immediate post-transplant time period based on early changes of liver-associated laboratory values including aspartate aminotransferase (AST), alanine aminotransferase (ALT), total bilirubin, and alkaline phosphatase.

\section{MATERIALS AND METHODS}

\section{Study design and population}

This retrospective study used a prospectively maintained institutional database of LT recipients at two tertiary care adacemic medical centers (Virginia Commonwealth University Mecical Center and Hunter Holmes McGuire VA Medical Center). Adult patients who underwent deceased donor LT between 2008 and 2019 were initially identified. Since this study was focused on patients with ABS, living donor LT (LDLT) recipients, patients with non-anastomotic strictures, those with ischemic cholangiopathy, and those with hepatic artery thrombosis were excluded. Patients with post-LT hepatocellular carcinoma (HCC) were also excluded.

These centers have a standardized operative technique for deceased donor LT which includes standard piggy-back reconstruction technique and end-to-end biliary reconstruction. All patients received the standard calcineurin inhibitor-based immunosuppression, mycophenolate mofetil 1,000-2,000 mg/day, and prednisone. Both mycophenolate mofetil and prednisone were tapered off within 4 months. None of these patients had normothermic perfusion. Per institutional protocols, LT recipients with concern for ABS or elevated liver enzymes should undergo extensive workup which typically includes an abdominal ultrasound with doppler, biochemical, and serological assessments for diseases including viral hepatitis, imaging studies including MRI, and careful review of medications.

All patients included in this study had baseline data collected at the time of LT and subsequently during follow-up every 3 to 6 months including MRI/MRCP. Laboratory data, medical history, biometric measurements, and operative parameters were recorded.

\section{Selection of patients with anastomotic biliary stricture}

LT recipients who underwent endoscopic retrograde cholangiopancreatography (ERCP) as well as MRCP were initially identified. Patients were excluded if the initial ERCP occurred prior to LT or if an alternative diagnosis such as choledocho- lithiasis or bile leak was identified. Among patients who underwent multiple ERCPs post-LT, only the initial ERCP was included in the analysis. Cholangiograms obtained during ERCP were assessed and a single cholangiogram was selected from each procedure for each patient as previously described [8].

The diagnosis of ABS was established by a blinded review of de-identified cholangiograms obtained during ERCP. Two expert interventional endoscopists independently reviewed cholangiograms as described previously $[6,8]$. ABS was defined as narrowing at the choledocho-choledochal anastomosis site with upstream dilation $[9,10]$. In the event of disagreement between the two interventional endoscopists regarding diagnosis of ABS, a review by a third expert endoscopist was undertaken. Notably, all patients selected for this study had also undergone MRCP for assessment of biliary anatomy.

\section{Rationale of control group selection}

Optimal controls were those who had undergone routine MRI at scheduled times after LT to better define normal biliary anatomy post-transplant. Among these patients, we selected patients who underwent LT for HCC since these patients routinely underwent scheduled MRI/MRCP post-LT to assess tumor recurrence [11]. Controls were utilized to define the expected post-LT anatomy. They were initially identified based on the following criteria: absence of elevated liver enzymes after LT, lack of clinical suspicion or radiological evidence of biliary stricture, and lack of ERCP after LT. These patients did not have any liver pathology post-LT. They underwent MRI only for surveillance. Surveillance MRI was performed every six months after LT till 18 months.

\section{Radiographic assessment}

"Normal" biliary anatomy expected after LT was assessed in the control population and compared with patients with ABS. The MRI performed at six months post-LT was considered the baseline exam. Subsequent MRIs performed every six months were used to assess temporal evolution of biliary anatomy. Each de-identified MRI/MRCP was independently reviewed by two expert radiologists who were blinded to clinical data.

The following parameters were evaluated: anastomosis length, diameter of anastomosis, and diameter of the common bile duct (CBD) at $1-\mathrm{cm}$ above and below the anastomosis. These diameters were measured by placing an electronic caliper on an MRCP image. Alternatively, T2-weighted half-Fourier single-shot turbo spin-echo images were used if MRCP images were degraded by artifacts. Mild waist-formation or narrowing of the end-to-end anastomosis and discrepancy between bile ducts of the donor and the recipient were used to localize the site of anastomosis. 'Anastomosis length' was defined as the length from the narrowest portion of the biliary anastomotic site identified by bile duct wasting on MRI to the start of upstream dilation. Corresponding measurements of radiologists were averaged. 


\section{Assessment of normal anatomy vs. anastomotic stricture}

We first attempted to define physiological changes in biliary anatomy expected in patients post-LT who did not develop biliary strictures. Hence, the biliary anatomy at baseline (i.e., MRI at six months) in control subjects was compared to that at follow-up MRI performed at 12 and 18 months post-LT using a paired Student's t-test. The distribution of biliary anatomy following LT was calculated at 5th, 25th, 50th, 75th, and 95th percentile on the empirical distribution of the data. The upper limit of normal biliary anatomy post-LT was set to be 95th percentile. The median length of the anastomosis, the median diameter of the anastomosis, and the median diameter of the bile duct at $1-\mathrm{cm}$ proximal and $1-\mathrm{cm}$ distal to the biliary anastomosis were calculated.

To determine the difference between normal post-LT biliary anatomy and $\mathrm{ABS}$, diameters of the $\mathrm{CBD}$ and anastomosis were compared between controls and patients with ABS using an unpaired Student's t-test.

\section{Assessment of lab abnormalities and strictures}

We hypothesized that the development of ABS could be detected in the immediate post-transplant time period based on early changes of AST, ALT, total bilirubin, and alkaline phosphatase. Therefore, we used separate Cox-regression models for each marker at day 7 after LT as an independent variable and development of biliary stricture as a dependent variable. Age, sex, cold ischemia time, and donation-after-circulatory death (DCD) allograft were used as covariates in the model. For sensitivity analysis, similar Cox-regression models at day 28 after LT were constructed.

\section{Statistical analyses}

Baseline variables in each group were reported as mean \pm standard deviation or median, interquartile range (IQR) for continuous variables, and frequencies and percentages for categorical variables. Continuous variables were compared between cases and controls using Student's t-test or Mann-Whitney $U$ test based on their distribution. Categorical variables were compared using Fisher's exact test. Regression analysis modelling is reported as hazard ratio (HR) with $95 \%$ confidence interval (CI). All statistical analyses were performed using IBM SPSS ver. 26 (IBM Corp., Armonk, NY, USA). A $p$-value $<0.05$ was considered statisticlly significant. The study protocol was approved by both Institutional Review Boards (no. HM20003025).

\section{RESULTS}

\section{Study cohort}

A total of 534 patients underwent deceased donor LT at our center from 2008 through 2019. Of these, 84 underwent ERCP post-LT. Finally, 57 patients ( 47 males) with a mean age of 53.9 \pm 8.3 years met the inclusion criteria for ABS. Corresponding- ly, 57 control patients (53 males) with mean age of $56.5 \pm 13.1$ years were selected based on inclusion and exclusion criteria defined above. All control patients had HCC. Preoperative total bilrubin levels were similar between the two groups. Overall, the leading etiology of cirrhosis requiring LT among patients was chronic hepatitis $\mathrm{C}$, followed by alcoholic liver disease and non-alcoholic steatohepatitis (Table 1).

\section{Normal biliary anatomy post-liver transplant}

Changes in the anatomy of CBD after LT were calculated by

Table 1. Baseline demographic characteristics of the study cohort

\begin{tabular}{|c|c|c|c|}
\hline Demographic & Control $(n=57)$ & $\operatorname{ABS}(n=57)$ & $p$-value \\
\hline Age in years & $56.5 \pm 13.1$ & $53.9 \pm 8.3$ & 0.22 \\
\hline Male & $53(93.0)$ & $47(82.5)$ & 0.08 \\
\hline Tobacco & $25(43.9)$ & $34(59.6)$ & 0.09 \\
\hline Race & & & 0.09 \\
\hline White & $36(63.2)$ & $43(75.4)$ & \\
\hline Black & $7(12.3)$ & $9(15.8)$ & \\
\hline Other & $14(24.6)$ & $5(8.8)$ & \\
\hline Etiology of cirrhosis & & & 0.06 \\
\hline Chronic hepatitis C & $31(54.4)$ & $27(47.4)$ & \\
\hline Alcoholic liver disease & $13(22.8)$ & $14(24.6)$ & \\
\hline Hepatitis B & $4(7.0)$ & $3(5.3)$ & \\
\hline Non alcoholic steatohepatitis & $9(15.8)$ & $6(10.5)$ & \\
\hline Primary biliary cholangitis & $0(0)$ & $2(3.5)$ & \\
\hline Other & $0(0)$ & $5(8.8)$ & \\
\hline \multicolumn{4}{|l|}{ Laboratory values at day 7} \\
\hline WBC $\left(10^{9} / \mathrm{L}\right)$ & 8.5 & 7.8 & 0.33 \\
\hline AST (units/L) & 47 & 68 & $<0.01$ \\
\hline ALT (units/L) & 139 & 130 & 0.58 \\
\hline Total bilirubin (mg/dL) & 1.4 & 1.9 & 0.03 \\
\hline Direct bilirubin (mg/dL) & 0.8 & 1.3 & $<0.01$ \\
\hline Alkaline phosphatase (IU/L) & 144 & 136 & 0.51 \\
\hline \multicolumn{4}{|l|}{ Laboratory values at day 28} \\
\hline AST (units/L) & 39 & 45 & 0.22 \\
\hline ALT (units/L) & 36 & 102 & $<0.01$ \\
\hline Total bilirubin (mg/dL) & 1.0 & 1.6 & 0.02 \\
\hline Direct bilirubin (mg/dL) & 0.6 & 1.1 & 0.02 \\
\hline Alkaline phosphatase (IU/L) & 132 & 208 & $<0.01$ \\
\hline \multicolumn{4}{|l|}{ Liver transplant parameters } \\
\hline Acute cellular rejection & $4(7.0)$ & $7(12.3)$ & 0.34 \\
\hline Cold ischemia time ( $\mathrm{h}: \mathrm{min})$ & $4: 50$ & $5: 45$ & $<0.01$ \\
\hline Preoperative bilirubin (mg/dL) & 10.6 & 12.1 & 0.85 \\
\hline $\mathrm{DCD}(\%)$ & $16(28.1)$ & $20(35.1)$ & 0.42 \\
\hline Total operative time $(\mathrm{h}: \mathrm{min})$ & $6: 08$ & $6: 25$ & 0.16 \\
\hline Intraoperative PRBC in liters & $1.5 \pm 1.2$ & $1.7 \pm 1.2$ & 0.53 \\
\hline Donor age in years & $36.6 \pm 5.6$ & $38.0 \pm 5.0$ & 0.21 \\
\hline
\end{tabular}

Values are presented as mean \pm standard deviation or number (\%). $A B S$, anastomotic biliary stricture; WBC, white blood cell; AST, aspartate aminotransferase; ALT, alanine aminotransferase; DCD, donation after circulatory death; PRBC, packed red blood cells. 
longitudinal assessment of serial MRI scans in control patients with pre-transplant HCC without known post-LT biliary disease. The median length of the anastomosis was $3.0 \mathrm{~mm}$ (IQR, $2.0-6.2 \mathrm{~mm}$ ) and corresponding diameter was $3.2 \mathrm{~mm}$ (IQR, $3.0-4.0 \mathrm{~mm}$ ) at six months. Correspondingly, the median diameter was $5.7 \mathrm{~mm}$ (IQR, $4.4-7.0 \mathrm{~mm})$ at $1-\mathrm{cm}$ proximal to the biliary anastomosis and $5.0 \mathrm{~mm}$ (IQR, $4.0-6.4 \mathrm{~mm}$ ) at $1-\mathrm{cm}$ distal to the biliary anastomosis at six months after LT. There were no significant changes in biliary anatomy over time between 6, 12, and 18 months (Fig. 1). To better define normal biliary anatomy, CBD parameters at 5th, 25th, 50th, 75th, and 95th percentiles are reported in Supplementary Table 1-3.

\section{Radiographic changes in anastomotic biliary stricture}

In the ABS group, the median time from LT to ABS was 322 days (IQR, 138-817 days). The mean diameter of the biliary anastomosis in the ABS group was significantly smaller than that of the control group $(2.47 \pm 1.32 \mathrm{~mm}$ vs. $3.38 \pm 1.05 \mathrm{~mm}$; $p<0.01)$. Notably, the diameter of CBD at $1-\mathrm{cm}$ proximal to the anastomosis was greater in the ABS group than in the con- trol group $(6.73 \pm 2.45 \mathrm{~mm}$ vs. $5.66 \pm 1.95 \mathrm{~mm} ; p=0.01)$. The diameter of CBD at $1-\mathrm{cm}$ distal to the biliary anastomosis and the length of the anastomosis were statistically similar between control and ABS groups (Fig. 2).

\section{Association of laboratory abnormalities and anastomotic biliary stricture}

Labs at day 7 and 28 were compared between ABS and control groups to assess their associations with ABS formation. Serum AST (68 vs. 47 units/L, $p<0.01$ ), total bilirubin $(1.9$ vs. $1.4 \mathrm{mg} / \mathrm{dL}$, $p=0.03$ ), and conjugated bilirubin ( 1.3 vs. $0.8 \mathrm{mg} / \mathrm{dL}, p<0.01$ ) were significantly higher in patients who developed ABS than in controls on day 7 after LT. Preoperative total bilurbin was decreased by $86.7 \%$ in the control group and $84.3 \%$ in the ABS group on day 7 post-LT.

Serum ALT (102 vs. 36 units/L; $p<0.01$ ), total bilirubin (1.6 vs. $1.0 \mathrm{mg} / \mathrm{dL} ; p=0.02)$, direct bilirubin (1.1 vs. $0.6 \mathrm{mg} / \mathrm{dL} ; p=0.02)$, and alkaline phosphatase (208 vs. 132 units/L; $p<0.01$ ) were significantly higher in patients who developed ABS than in controls on postoperative day 28 (Table 1). Preoperative to-
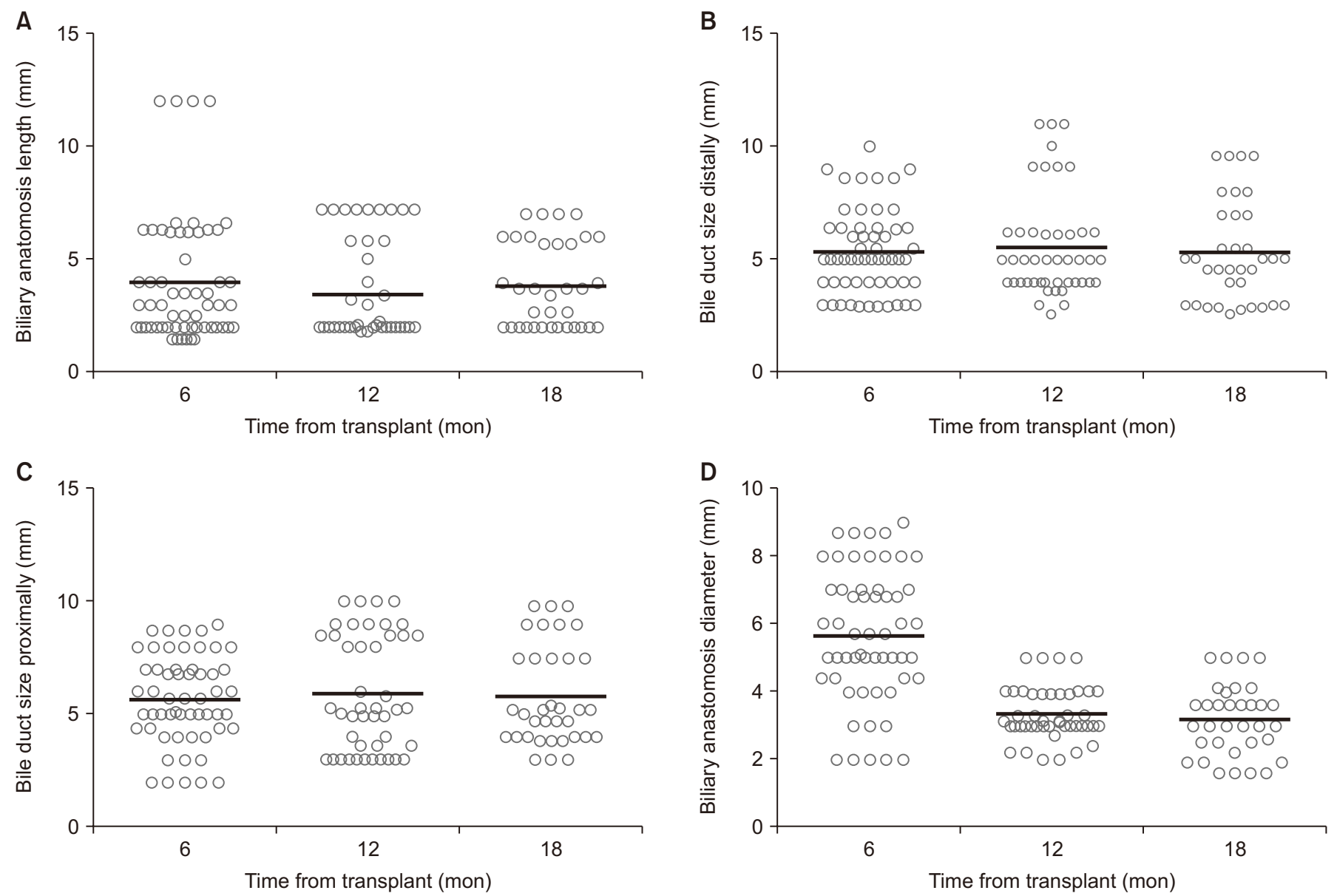

Fig. 1. Normal anatomical changes in measurements of biliary anastomosis length (A), common bile duct at $1 \mathrm{~cm}$ distal to the anastomosis (B), common bile duct size at $1 \mathrm{~cm}$ proximal to the anastomosis (C), and the caliber of bile duct anastomosis (D) over 18 months post-liver transplant. 
- Control cohort

- Anastomotic stricture cohort

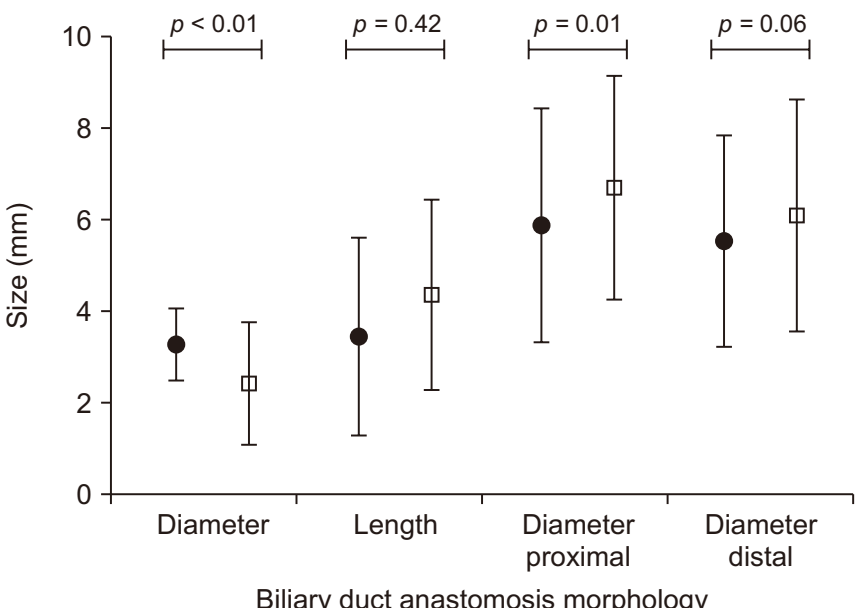

Fig. 2. Comparison of biliary morphology between patients with post liver transplant anastomotic biliary strictures and controls. The length of anastomosis, diameter of the anastomosis, and proximal and distal biliary segments were compared. Anatomoy for the subject group was recorded at the time of presentation and diagnoses of anastomotic biliary stricture while that for the control group was recorded at 6-month post-transplant.

tal bilurbin was decreased by $90.6 \%$ in the control group and $86.8 \%$ in the ABS group on day 28 post-LT.

The strength of the association between elevated liver enzymes and ABS formation was evaluated using adjusted and unadjusted Cox-regression analysis on postoperative day 7 and day 28. Labs on day 7 demonstrated statistically significant associations with the likelihood of developing ABS: serum AST (HR: 1.014, 95\% CI: 1.008-1.020; $p=0.001$ ), total bilirubin (HR: $1.292,95 \%$ CI: $1.100-1.517 ; p=0.002$ ), and conjugated bilirubin
(HR: $1.467,95 \%$ CI: $1.216-1.768 ; p=0.001)$.These associations remained statistically significant after adjusting for age, sex, cold ischemia time, and DCD donor allograft (Table 2).

Unadjusted Cox-regression analysis demonstrated statistically significant associations of ABS formation with labs on day 28: serum alkaline phosphatase (HR, 1.005, 95\% CI: 1.003-1.007; $p=0.001$ ), total bilirubin (HR: 1.233 , 95\% CI: $1.110-1.369 ; p=0.001$ ), and conjugated bilirubin (HR: 1.272 , 95\% CI: $1.126-1.437 ; p=0.001$ ). Serum AST (HR: 1.001, 95\% CI: $0.999-1.004 ; p=0.227$ ) was not significantly different between the two patients groups at day 28, whereas serum ALT (HR: 1.004, 95\% CI: 1.002-1.006; $p=0.001$ ) was. These associations remained statistically significant after adjusting for age, sex, cold ischemia time, and DCD donation (Table 2).

\section{DISCUSSION}

Accurately predicting post-LT ABS in the early postoperative time-period remains a challenge and precludes early interventions to prevent adverse outcomes. Notably, it is important to differentiate between a stricture that can obstruct the flow of bile or raise liver enzymes and usual morphological changes that are expected to occur with fibrotic healing at the anastomosis $[6,12]$. This current study scientifically defined post-LT ABS and used postoperative liver enzyme elevation to assess likelihood of subsequent stricture formation.

This study demonstrates that changes in liver enzymes early after LT are associated with subsequent development of ABS. This suggests that inciting events can occur peri-operatively, although they might take time to become clinically evident. While the etiopathogenesis of the process is not entirely clear, possible mechanisms include surgical injury, localized ischemic event, and sub-optimal quality of donated organ [13]. Other investigators have also suggested that elevation of liver

Table 2. Cox-regression analysis for association between early laboratory values and development of anastomotic biliary strictures

\begin{tabular}{|c|c|c|c|c|}
\hline Laboratory value & Unadjusted HR (95\% Cl) & $p$-value & Adjusted HR $(95 \% \mathrm{Cl})^{\mathrm{a}}$ & $p$-value \\
\hline AST & $1.014(1.008-1.020)$ & 0.001 & $1.014(1.009-1.020)$ & 0.001 \\
\hline Total bilirubin & $1.292(1.100-1.517)$ & 0.002 & $1.304(1.110-1.531)$ & 0.001 \\
\hline Direct bilirubin & $1.467(1.216-1.768)$ & 0.001 & $1.482(1.224-1.794)$ & 0.001 \\
\hline Alkaline phosphatase & $0.999(0.994-1.003)$ & 0.563 & $0.998(0.993-1.003)$ & 0.342 \\
\hline AST & $1.001(0.999-1.004)$ & 0.227 & $1.002(0.999-1.004)$ & 0.182 \\
\hline ALT & $1.004(1.002-1.006)$ & 0.001 & $1.004(1.002-1.006)$ & 0.001 \\
\hline Total bilirubin & $1.233(1.110-1.369)$ & 0.001 & $1.189(1.063-1.329)$ & 0.002 \\
\hline Direct bilirubin & $1.272(1.126-1.437)$ & 0.001 & $1.220(1.071-1.390)$ & 0.003 \\
\hline Alkaline phosphatase & $1.005(1.003-1.007)$ & 0.001 & $1.005(1.003-1.007)$ & 0.005 \\
\hline
\end{tabular}

$\mathrm{HR}$, hazards ratio; $\mathrm{Cl}$, confidence interval; $\mathrm{AST}$, aspartate aminotransferase; $\mathrm{ALT}$, alanine aminotransferase.

${ }^{\text {a) }}$ Adjusted for age, sex, cold ischemia time, and donation after circulatory death donor allograft. 
associated enzymes in the early postoperative period might be associated with post-LT stricture formation $[12,14,15]$. However, these studies had methodological limitations including small number of patients [15], lack of a control group [14], and lack of blinded confirmation of stricture formation [12]. To the best of our knowledge, this is the first study to scientifically define a stricture with blinded reviews of cholangiograms, to compare imaging findings to a control population, and to assess the strength of association of stricture formation with early elevation of liver associated enzymes.

Abnormalities in the hepatic panel are often the first clues into the presence of ABS. However, these are common and non-specific. They can occur due to several etiologies such as ischemia, rejection, recurrence of liver disease, de novo non-alcoholic fatty liver disease [16], and infections. We report significant associations between ABS formation and elevated levels of total bilirubin and direct bilirubin levels at day 7 and day 28 postLT. Another study has also noted the association between stricture formation and early elevation of bilirubin levels. However, that study was restricted to patients who received DCD liver allografts [17] and limited the assessment to 7 days post-LT.

Notably, we also report an association between ABS formation and elevation in AST at day 7 post-LT and ALT at day 28 post-LT. ALT has been shown to be a more specific marker of hepatocyte injury than AST since AST is also present in cardiac and skeletal muscle, kidney, brain, and erythrocytes $[18,19]$. Furthermore, AST is present within metabolically active mitochondria of hepatocytes. AST is also concentrated in Zone 3 of the hepatic acinus which is more susceptible to ischemia encountered during surgery compared to Zone $1[20,21]$. We speculate that labs on day 7 post-LT can indicate early surgical insult from hypoperfusion, leading to transient elevations of serum AST. In contrast, labs on day 28 post-LT could represent sequelae of ductal injury with more hepatocyte-specific serum ALT changes.

Lack of 'normal' measures of biliary anatomy following LT has limited the diagnostic performance of radiological testing in $\mathrm{LT}$ recipients $[22,23]$. In the present study, we used a cohort of low-risk patients without evidence of biliary strictures to define normal biliary measurements. Detailed measurements of the biliary tree suggest that diameters of biliary anastomosis and $\mathrm{CBD}$ at $1-\mathrm{cm}$ proximal to the biliary anastomosis are the most helpful measures when assessing the presence of ABS. In comparison, the anastomotic length and the caliber of the CBD at $1-\mathrm{cm}$ distal to the biliary anastomosis possibly represent normal variations of post-surgical anatomy. These measurements can potentially be readily incorporated into algorithm-based testing or artificial intelligence platforms to improve diagnostic performance.

This study has several strengths, including a robust consensus definition of biliary strictures, a blinded and detailed review of radiological parameters to establish normal biliary anatomy post-LT, and longitudinal measurements to demon- strate time-dependent changes in biliary anatomy. However, this study is limited by its two-center retrospective design, small sample size, and lack of a validation cohort. To overcome the limitation of the sample size, all radiographic parameters were plotted on a histogram to ensure normal distribution. LDLTs were excluded from the present study because the incidence of ABS in those with LDLTs was disproportionally higher that that in deceased donor LT. We suspect that the underlying pathophysiology might be different, which could impact the current proof-of-concept study. Finally, subjects in the control arm did have a normal hepatic panel. However, they did not undergo definitive testing (i.e., ERCP) to exclude the presence of biliary stricture. While this might be a potential limitation of the retrospective study design, subjecting patients to invasive and unnecessary testing would be difficult to ethically justify since this testing would not offer any clinical benefit to patients. In the present study, several exclusion criteria were used to ensure that patients in the control arm did not have ABS.

This study provides novel data regarding the potential pathophysiology of ABS that can be better studied in a prospective cohort interrogating how an early insult after LT can predispose patients to develop ABS. It also provides necessary data to design future studies that are adequately powered using best-practice biomarker validation statistical designs. Finally, artificial intelligence platforms can use data of this study to refine the diagnostic accuracy of imaging studies [24]. However, more robust multi-center, retrospective studies are needed to develop a diagnostic algorithm followed by future prospective studies to validate this model to improve early detection and management of biliary strictures. These validation models could have the potential to detect high-risk patients for the development of $\mathrm{ABS}$ in order to employ preventative (i.e., anti-fibrotic medications) strategies to prevent biliary strictures medically. The current study encourages more focused research studies to better define the clinical history and pathophysiology of ABS.

In conclusion, morphological changes at the site of biliary anastomosis can be distinguished from post-LT ABS. Early elevation of post-LT laboratory values is strongly associated with future risk of developing ABS. Using these data, prospective studies can be designed to develop algorithm-based models for predicting post-LT ABS.

\section{SUPPLEMENTARY DATA}

Supplementary data related to this article can be found at https://doi.org/10.14701/ahbps.21-103.

\section{FUNDING}

None. 


\section{CONFLICT OF INTEREST}

Mohammad S. Siddiqui: Grant support Zydus, Pfizer consultant, Divyanshoo Rai Kohli: Research funding from Olympus, Tilak Shah: CSA Medical research advisory board, Allergen research support, Research support Lucid. Except for that, no potential conflict of interest relevant to this article was reported.

\section{ORCID}

Matthew Fasullo, https://orcid.org/0000-0003-3787-7626 Priyanush Kandakatla, https://orcid.org/0000-0003-3411-6637 Reza Amerinasab, https://orcid.org/0000-0002-6680-5667 Divyanshoo Rai Kohli, https://orcid.org/0000-0002-7801-0044 Tilak Shah, https://orcid.org/0000-0003-4818-2571 Samarth Patel, https://orcid.org/0000-0003-1531-6959 Chandra Bhati, https://orcid.org/0000-0002-4333-5551 Doumit Bouhaidar, https://orcid.org/0000-0002-8470-1300 Mohammad S. Siddiqui, https://orcid.org/0000-0001-7392-7489 Ravi Vachhani, https://orcid.org/0000-0001-8824-0196

\section{AUTHOR CONTRIBUTIONS}

Conceptualization: MF, MSS. Data curation: MF, PK, RA, SP. Methodology: MF, TS, DB, MSS, RV. Visualization: MF, DRK, CB. Writing - original draft: MF, TS. Writing - review \& editing: MF, TS, MSS.

\section{REFERENCES}

1. Kohli DR, Shah TU, BouHaidar DS, Vachhani R, Siddiqui MS. Significant infections in liver transplant recipients undergoing endoscopic retrograde cholangiography are few and unaffected by prophylactic antibiotics. Dig Liver Dis 2018;50:1220-1224.

2. Kohli DR, Harrison ME, Mujahed T, Fukami N, Faigel DO, Pannala R, et al. Outcomes of endoscopic therapy in donation after cardiac death liver transplant biliary strictures. HPB (Oxford) 2020;22:979-986.

3. Kohli DR, Desai MV, Kennedy KF, Pandya P, Sharma P. Patients with post-transplant biliary strictures have significantly higher rates of liver transplant failure and rejection: a nationwide inpatient analysis. J Gastroenterol Hepatol 2021;36:2008-2014.

4. Sharma S, Gurakar A, Jabbour N. Biliary strictures following liver transplantation: past, present and preventive strategies. Liver Transpl 2008;14:759-769.

5. Rerknimitr R, Sherman S, Fogel EL, Kalayci C, Lumeng L, Chalasani $\mathrm{N}$, et al. Biliary tract complications after orthotopic liver transplantation with choledochocholedochostomy anastomosis: endoscopic findings and results of therapy. Gastrointest Endosc 2002;55:224-231.

6. Kohli DR, Vachhani R, Shah TU, BouHaidar DS, Siddiqui MS. Diagnostic accuracy of laboratory tests and diagnostic imaging in detecting biliary strictures after liver transplantation. Dig Dis Sci 2017;62:1327-1333.
7. Kochhar G, Parungao JM, Hanouneh IA, Parsi MA. Biliary complications following liver transplantation. World J Gastroenterol 2013;19:2841-2846

8. Kohli DR, Pannala R, Crowell MD, Fukami N, Faigel DO, Aqel BA, et al. Interobserver agreement for classifying post-liver transplant biliary strictures in donation after circulatory death donors. Dig Dis Sci 2021;66:231-237.

9. Simoes P, Kesar V, Ahmad J. Spectrum of biliary complications following live donor liver transplantation. World J Hepatol 2015;7:18561865.

10. Tabibian JH, Girotra M, Yeh HC, Singh VK, Okolo PI III, Cameron $\mathrm{AM}$, et al. Risk factors for early repeat ERCP in liver transplantation patients with anastomotic biliary stricture. Ann Hepatol 2015;14:340347.

11. Lucey MR, Terrault N, Ojo L, Hay JE, Neuberger J, Blumberg E, et al. Long-term management of the successful adult liver transplant: 2012 practice guideline by the American Association for the Study of Liver Diseases and the American Society of Transplantation. Liver Transpl 2013;19:3-26

12. Nair S, Lingala S, Satapathy SK, Eason JD, Vanatta JM. Clinical algorithm to guide the need for endoscopic retrograde cholangiopancreatography to evaluate early postliver transplant cholestasis. Exp Clin Transplant 2014;12:543-547.

13. Rao HB, Prakash A, Sudhindran S, Venu RP. Biliary strictures complicating living donor liver transplantation: problems, novel insights and solutions. World J Gastroenterol 2018;24:2061-2072.

14. Woo YS, Lee KH, Lee KT, Lee JK, Kim JM, Kwon CHD, et al. Postoperative changes of liver enzymes can distinguish between biliary stricture and graft rejection after living donor liver transplantation: a longitudinal study. Medicine (Baltimore) 2017;96:e6892.

15. Ben-Ari Z, Weiss-Schmilovitz H, Sulkes J, Brown M, Bar-Nathan N, Shaharabani E, et al. Serum cholestasis markers as predictors of early outcome after liver transplantation. Clin Transplant 2004;18:130-136.

16. Bhati C, Idowu MO, Sanyal AJ, Rivera M, Driscoll C, Stravitz RT, et al. Long-term outcomes in patients undergoing liver transplantation for nonalcoholic steatohepatitis-related cirrhosis. Transplantation 2017;101:1867-1874.

17. Kohli DR, Harrison ME, Adike AO, El Kurdi B, Fukami N, Faigel DO, et al. Predictors of biliary strictures after liver transplantation among recipients of dcd (donation after cardiac death) grafts. Dig Dis Sci 2019;64:2024-2030.

18. Wroblewski F. The clinical significance of alterations in transaminase activities of serum and other body fluids. Adv Clin Chem 1958;1:313351.

19. Hall P, Cash J. What is the real function of the liver 'function' tests? Ulster Med J 2012;81:30-36.

20. Kamimoto Y, Horiuchi S, Tanase S, Morino Y. Plasma clearance of intravenously injected aspartate aminotransferase isozymes: evidence for preferential uptake by sinusoidal liver cells. Hepatology 1985;5:367-375.

21. Turlin B, Slapak GI, Hayllar KM, Heaton N, Williams R, Portmann B. Centrilobular necrosis after orthotopic liver transplantation: a longitudinal clinicopathologic study in 71 patients. Liver Transpl Surg 
1995;1:285-289.

22. Girometti R, Como G, Bazzocchi M, Zuiani C. Post-operative imaging in liver transplantation: state-of-the-art and future perspectives. World J Gastroenterol 2014;20:6180-6200.

23. Thethy S, Thomson BNJ, Pleass H, Wigmore SJ, Madhavan K, Akyol
M, et al. Management of biliary tract complications after orthotopic liver transplantation. Clin Transplant 2004;18:647-653.

24. Le Berre C, Sandborn WJ, Aridhi S, Devignes MD, Fournier L, Smaïl-Tabbone M, et al. Application of artificial intelligence to gastroenterology and hepatology. Gastroenterology 2020;158:76-94.e2. 